ARTICLE

Received 12 Aug 2015 | Accepted 9 Oct 2015 | Published 16 Nov 2015

DOI: $10.1038 /$ ncomms 9867

OPEN

\title{
The full repertoire of Drosophila gustatory receptors for detecting an aversive compound
}

\author{
Jaewon Shim ${ }^{1,2, \star}$, Youngseok Lee ${ }^{3, \star}$, Yong Taek Jeong ${ }^{1, \star}$, Yonjung Kim ${ }^{4, \star}$, Min Goo Lee ${ }^{4}$, \\ Craig Montell ${ }^{5} \&$ Seok Jun Moon ${ }^{1}$
}

The ability to detect toxic compounds in foods is essential for animal survival. However, the minimal subunit composition of gustatory receptors required for sensing aversive chemicals in Drosophila is unknown. Here we report that three gustatory receptors, GR8a, GR66a and GR98b function together in the detection of L-canavanine, a plant-derived insecticide. Ectopic co-expression of Gr8a and Gr98b in Gr66a-expressing, bitter-sensing gustatory receptor neurons (GRNs) confers responsiveness to L-canavanine. Furthermore, misexpression of all three Grs enables salt- or sweet-sensing GRNs to respond to L-canavanine. Introduction of these Grs in sweet-sensing GRNs switches L-canavanine from an aversive to an attractive compound. Co-expression of GR8a, GR66a and GR98b in Drosophila S2 cells induces an L-canavanine-activated nonselective cation conductance. We conclude that three GRs collaborate to produce a functional L-canavanine receptor. Thus, our results clarify the full set of GRs underlying the detection of a toxic tastant that drives avoidance behaviour in an insect.

\footnotetext{
${ }^{1}$ Department of Oral Biology, BK21 PLUS Project Yonsei University College of Dentistry, Yonsei-ro 50-1, Seodaemun-gu, Seoul 120-752, Korea. ${ }^{2}$ Division of Functional Food Research, Korea Food Research Institute, Seongnam, Gyeonggi-do 463-746, Korea. ${ }^{3}$ Department of Bio and Fermentation Convergence Technology, Kookmin University, Seoul 136-702, Korea. ${ }^{4}$ Department of Pharmacology, Brain Korea 21 PLUS project for Medical Sciences Yonsei University College of Medicine, Yonsei-ro 50-1, Seodaemun-gu, Seoul 120-752, Korea. ${ }^{5}$ Department of Molecular, Cellular, and Developmental Biology, Neuroscience Research Institute, University of California, Santa Barbara, California 93106, USA. ${ }^{\star}$ These authors contributed equally to this work. Correspondence and requests for materials should be addressed to C.M. (email cmontell@lifesci.ucsb.edu) or to S.J.M. (email sjmoon@yuhs.ac).
} 
T aste is critical for evaluating food quality. Dedicated sensory cells are devoted to each taste modality, sending sensory information to higher brain centres that direct either attraction or aversion ${ }^{1}$. Because many bitter and other avoidance compounds are toxic, rapid and accurate detection of these compounds is an important defense in many herbivorous animals.

In Drosophila, several types of membrane proteins participate in the perception of aversive chemicals. At least two transient receptor potential (TRP) channels, TRPA1 and TRPL, function in the sensation of deterrent compounds ${ }^{2-4}$. However, the detection of most aversive tastants are thought to be accomplished through members of the gustatory receptor (GR) family, which encodes 68 proteins ${ }^{5-8}$. Fly GRs are unrelated to mammalian taste receptors, which are G-protein coupled receptors, but they are distantly related to the Drosophila olfactory receptors (ORs) ${ }^{7,9-14}$. Heteromeric OR complexes comprise odorant-gated-cation channels ${ }^{15-17}$, and it is reported that insect fructose receptors consist of single GR subunits, which form nonselective cation channels $^{18}$.

The Drosophila GRs that respond to noxious compounds consist of multiple subunits. Based on in vivo loss-of-function studies, three GRs are broadly tuned (GR32a, GR33a and GR66a) and function in the detection of a wide range of avoidance compounds $^{19-21}$. In addition, other GRs, such as GR8a, GR47a and GR93a, are narrowly tuned and required for sensing L-canavanine, strychnine and caffeine, respectively ${ }^{22-24}$.

$\mathrm{L}$-canavanine is a plant-derived analogue of the amino acid L-arginine ${ }^{25-27}$, and ingestion of this compound is lethal to fruit flies and many other insects because it incorporates into proteins in place of $\mathrm{L}$-arginine. We previously reported that GR8a and GR66a are required for L-canavanine detection ${ }^{22}$. However, coexpression of Gr8a and Gr66a in sweet-sensing GRNs does not confer responsiveness to L-canavanine. Currently, the minimum subunit composition of the L-canavanine receptor or any of the other GR complexes that respond to aversive compounds are unknown. It is also unclear if these heteromultimeric GRs are cation channels.

In this study, we perform an RNA interference (RNAi) screen to identify the complete set of receptors involved in L-canavanine detection. As expected, knockdown of Gr8a and Gr66a impairs Lcanavanine avoidance behaviour. In addition, we find that suppressing expression of one additional gene ( $\mathrm{Gr} 98 \mathrm{~b})$ disrupts L-canavanine avoidance. We deleted $G r 98 b$, which eliminates the behavioural repulsion and action potentials in response to Lcanavanine. Introduction of Gr8a, Gr66a and Gr98b together in sweet-sensing GRNs or low salt-sensing GRNs, endows these cells with the ability to respond to L-canavanine. Moreover, ectopic expression of these GRs in sweet-sensing GRNs switches the flies' innate L-canavanine aversion to attraction. Ectopic expression of Gr8a, Gr66a and Gr98b in S2 tissue culture cells confers L-canavanine-dependent currents. Our findings define the first heteromultimeric GR complex that is required and sufficient for conferring sensitivity to an aversive compound.

\section{Results}

Screening for receptors required for L-canavanine detection. Our previous findings show that GR8a and GR66a are required but not sufficient for L-canavanine detection ${ }^{22}$. Therefore, we performed an RNAi screen to address a potential requirement for other GRs, by interrogating the full set of 58 available $U A S-G r$ RNAi lines. We also knocked down 13 genes encoding ionotropic receptors (IRs) that were expressed in GRNs ${ }^{28,29}$. We crossed these RNAi lines to flies that expressed Dicer (UAS-Dcr2) and the Gr33a-GAL4 driver, which is expressed in GRNs that respond to aversive compounds ${ }^{21}$. All of the progeny were viable and appeared healthy.

To assess L-canavanine avoidance, we performed two-way choice behavioural assays. Given a choice between $1 \mathrm{mM}$ sucrose

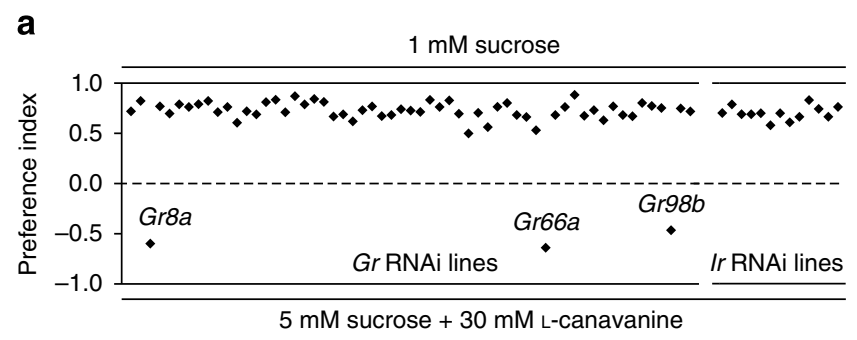

b
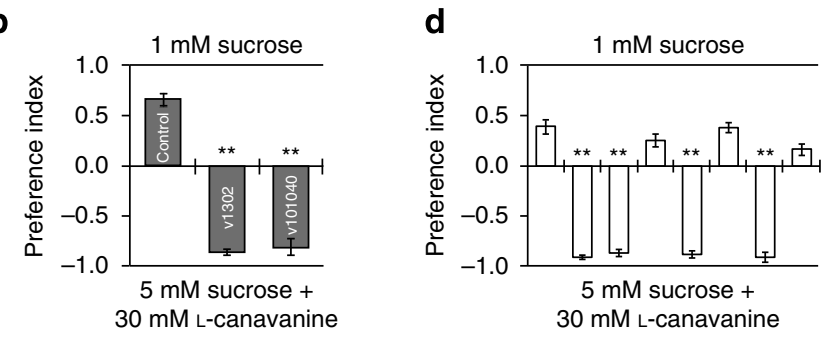

C

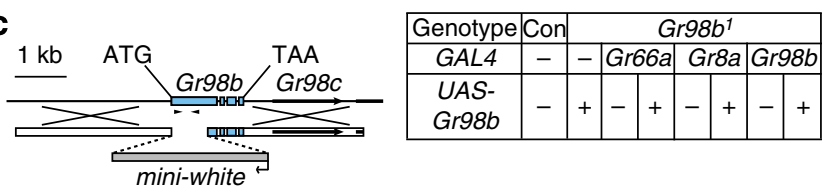

e

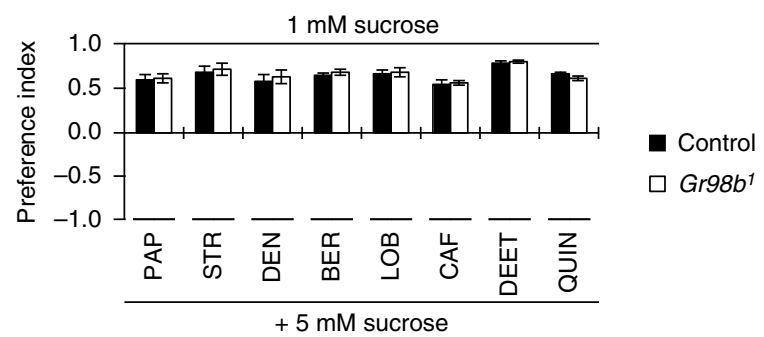

Figure 1 | Identification of taste receptors required for L-canavanine avoidance. (a) An RNAi screen of 58 UAS-Gr RNAi lines and 13 UAS-Ir RNAi lines for defects in L-canavanine avoidance. We drove expression of the RNAi lines using the Gr33a-GAL4, and included UAS-Dcr2 (Dicer2) to improve the efficacy of the RNAi. The dashed line indicates no preference. See the Methods section (fly stocks) for the list of stocks screened. (b) Two-way choice assays after knocking down Gr98b using two different RNAi lines. The control consisted of UAS-Dcr2;Gr33a-GAL4 flies without the RNAi transgenes. RNAi stock numbers (VDRC) are indicated within the bars. $n=5$ for each genotype. ${ }^{\star \star} P<0.01$ (analysis of variance (ANOVA) with post hoc Tukey test). (c) Cartoon showing the strategy for creating the Gr98 $a^{7}$ allele by ends-out homologous recombination. The arrowheads indicate the genomic PCR primers used to confirm the Gr98b deletion. A 543 bp band present in control flies was absent in $\mathrm{Gr} 98 b^{\top}$. (d) Two-way choice assays to test whether Gr98 $b^{7}$ displayed a deficit in L-canavanine avoidance. To test for rescue of the $\mathrm{Gr} 98 b^{7}$ phenotype, we expressed the Gr98b cDNA in the Gr98b background using the Gr66a-GAL4, the Gr8a-GAL4 and the Gr98b-GAL4. $n=5$ for each genotype. ${ }^{* \star} P<0.01$ (ANOVA with post hoc Tukey test). (e) Two-way choice assays to test for avoidance of $\mathrm{Gr} 98 b^{7}$ flies in response to the indicated bitter chemicals. The flies were given a choice between $1 \mathrm{mM}$ sucrose and $5 \mathrm{mM}$ sucrose plus the following aversive compounds: $0.5 \mathrm{mM}$ papaverine (PAP), $0.5 \mathrm{mM}$ strychnine (STR), $0.1 \mathrm{mM}$ denatonium (DEN), $0.05 \mathrm{mM}$ berberine (BER), $0.1 \mathrm{mM}$ lobeline (LOB), $5 \mathrm{mM}$ caffeine (CAF), $0.2 \% \mathrm{~N}, \mathrm{~N}$-diethyl-mtoluamide (DEET), and $0.5 \mathrm{mM}$ quinine (QUIN). $n=4-7$ for each genotype. All data are mean \pm s.e.m. 

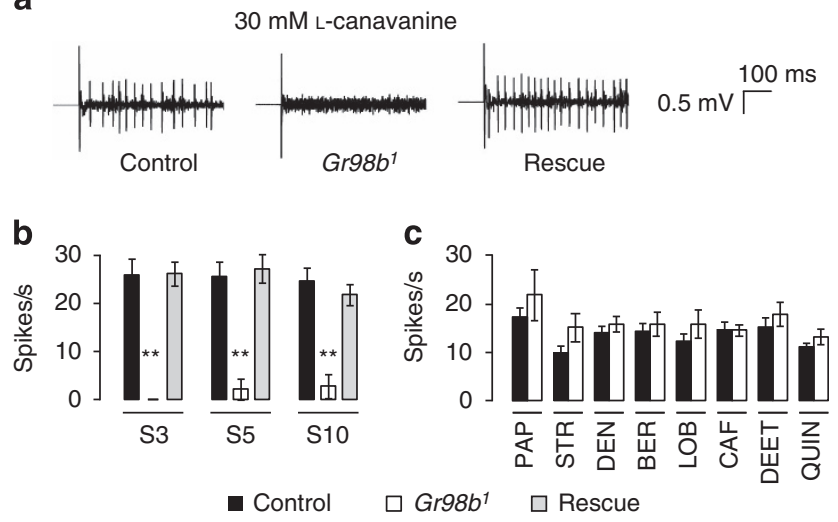

Figure 2 | Dependence of L-canavanine induced action potentials on Gr98b. (a) Action potentials elicited in $\mathrm{S} 6$ sensilla of control ( $\left.w^{1118}\right), \mathrm{Gr} 98 \mathrm{~b}^{1}$ and rescue flies (Gr66a-GAL4/UAS-Gr98b;Gr98b') in response to $30 \mathrm{mM}$ L-canavanine. (b) Mean frequencies of action potentials upon exposure to $30 \mathrm{mM}$ L-canavanine. Indicated are the genotypes and sensilla tested (S3, S5 and S10), $n=10-12$ for each genotype. (c) Mean frequencies of action potential induced in $\mathrm{S} 6$ sensilla in response to the indicated bitter chemicals (1 mM PAP, $1 \mathrm{mM}$ STR, $1 \mathrm{mM}$ DEN, $0.1 \mathrm{mM}$ BER, $1 \mathrm{mM} \mathrm{LOB}, 10 \mathrm{mM}$ CAF, $0.2 \%$ DEET and $1 \mathrm{mM}$ QUIN). $n=10-21$ for each genotype. All data are mean \pm s.e.m. ${ }^{\star \star} P<0.01$ (analysis of variance with post hoc Tukey test). and $5 \mathrm{mM}$ sucrose mixed with $30 \mathrm{mM}$ L-canavanine, wild-type flies strongly avoid the higher sugar laced with L-canavanine ${ }^{22}$. As expected, knockdown of either Gr8a or Gr66a dramatically reduced L-canavanine avoidance ${ }^{22}$ (Fig. 1a). In addition, we found that RNAi-mediated suppression of one other receptor $(\mathrm{Gr} 98 \mathrm{~b})$ also reduced L-canavanine avoidance dramatically (Fig. 1a; v101040 line). Introduction of all other UAS-Gr or UAS-Ir RNAi lines had no impact on L-canavanine avoidance (Fig. 1a). We tested an additional UAS-Gr98b RNAi line (v1302), which produced the same phenotype as the first line (v101040; Fig. 1b). Thus, GR98b was an additional candidate receptor critical for detecting L-canavanine.

Mutation of Gr98b impaired L-canavanine detection. To confirm a role for $G r 98 b$ for L-canavanine repulsion, we generated a mutation by ends-out homologous recombination (Fig. 1c). The $G r 98 b^{1}$ mutation deleted the region encoding the N-terminal 233 out of 403 residues. The mutant flies were homozygous viable and fertile. Consistent with the RNAi experiments, the $G r 98 b^{1}$ flies failed to avoid L-canavanine (Fig. 1d). We fully rescued L-canavanine avoidance in the $G r 98 b^{1}$ flies by expressing a wild-type $G r 98 b$ transgene (UAS-Gr98b) under the control of the Gr66a (Gr66aGAL4), the Gr8a (Gr8a-GAL4) or the Gr98b promoter ( $G r 98 b-$ GAL4; Fig. 1d). L-canavanine-induced action potentials were also
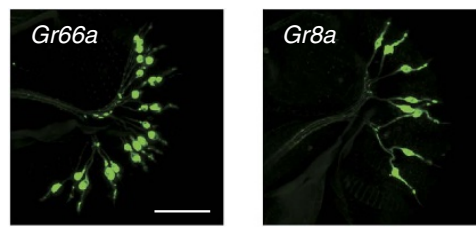

b
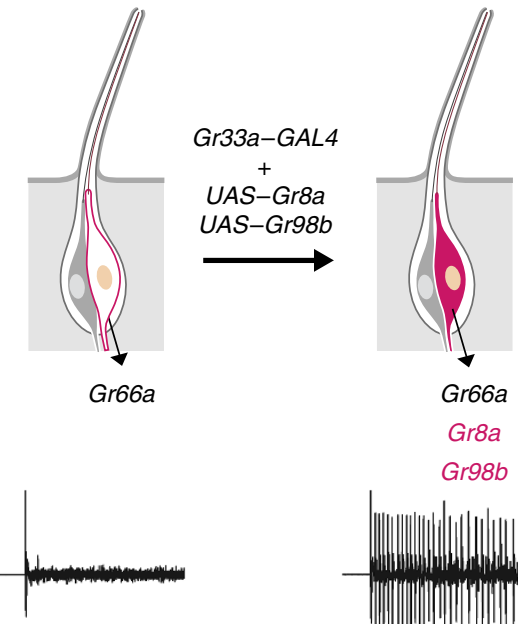

$0.5 \mathrm{mV} \Gamma^{100} \mathrm{~ms}$
Gr8a

Gr33a-GAL4

UAS-Gr98

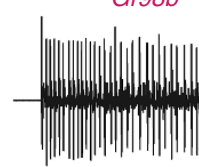

- UAS-Gr8a,UAS-Gr98b
UAS-Gr8a
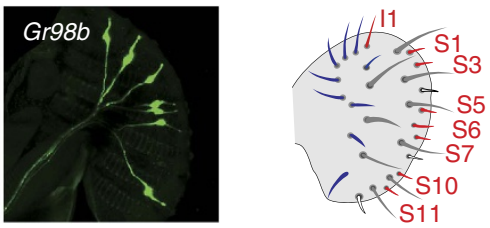

- Gr66a only

- Gr8a + Gr66a + Gr98b

C
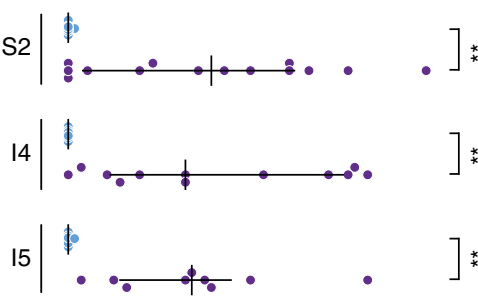

$16 \mid\left[\begin{array}{l}\ldots+\ldots \\ \hdashline \ldots *\end{array}\right]$
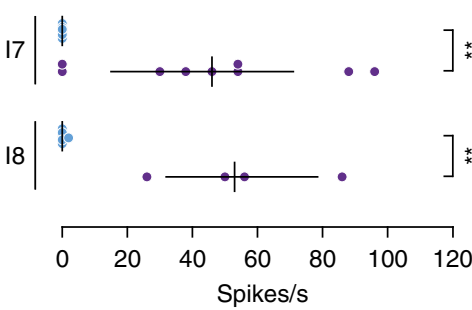

Figure 3 | Ectopic Gr8a and Gr98b expression in bitter-sensing GRNs conferred L-canavanine sensitivity. (a) Expression patterns of the Gr8a, the Gr66a and the Gr98b reporters as well as a cartoon indicating the location of Gr8a-, Gr66a- and Gr98b-expressing sensilla in the labellum. The scale bar represents $50 \mu \mathrm{m}$. (b) Ectopic expression of Gr8a and Gr98b in bitter-sensing GRNs (I-type sensilla) conferred L-canavanine sensitivity to sensilla that did not normally respond to L-canavanine. A schematic representation of a sensilla depicting the ectopic expression experiment (above) and representative traces (below) evoked by 30 mM L-canavanine from 14 sensilla of control (UAS-Gr8a,UAS-Gr98b) and UAS-Gr8a,UAS-Gr98b;Gr33a-GAL4 flies. (c) Response frequencies evoked by $30 \mathrm{mM}$ L-canavanine after ectopic expression of Gr8a and Gr98b in the indicated sensilla. UAS-Gr8a,UAS-Gr98b flies were the negative controls. $n=4-14$. ${ }^{\star \star} P<0.01$ (Mann-Whitney $U$-test). Medians and quartiles are shown. 
abolished in $G r 98 b^{1}$ flies, and the defect was rescued by expressing the wild-type Gr98b transgene (UAS-Gr98b) using the Gr66aGAL4 (Fig. 2a,b). In contrast to the effects on sensing L-canavanine, $G r 98 b^{1}$ flies displayed robust aversion and electrophysiological responses to papaverine, strychnine, denatonium, berberine, lobeline, caffeine, N,N-diethyl-m-toluamide (DEET) and quinine (Figs 1e and 2c). These data indicated that GR98b was required for the detection of L-canavanine, and was narrowly tuned.

Endowing L-canavanine responsiveness to bitter-sensing GRNs. The major taste organ of the fly, the labellum, is decorated with gustatory bristles (sensilla) that fall into three classes based on length and position: long (L), intermediate (I) and short $(\mathrm{S})^{1}$. Gr66a is widely expressed in bitter-sensing GRNs of S-type and I-type sensilla ${ }^{30-32}$, whereas Gr8a expression is limited to the subset of Gr66a-expressing GRNs that respond to L-canavanine ${ }^{22}$. To determine the expression pattern of $G r 98 b$, we examined GFP staining in Gr98b-GAL4;UAS-mCD8::GFP flies. We detected Gr98b-GAL4 reporter expression in GRNs of I1, S1, S3, S5, S6, S7, S10 and S11 sensilla (Fig. 3a; Supplementary Table 1). Consistent with a role in sensing L-canavanine, this included all of the sensilla that responded to L-canavanine ${ }^{22}$, and the S-type sensilla that contained GRNs that expressed the Gr8a-GAL4 (refs 22,30). a

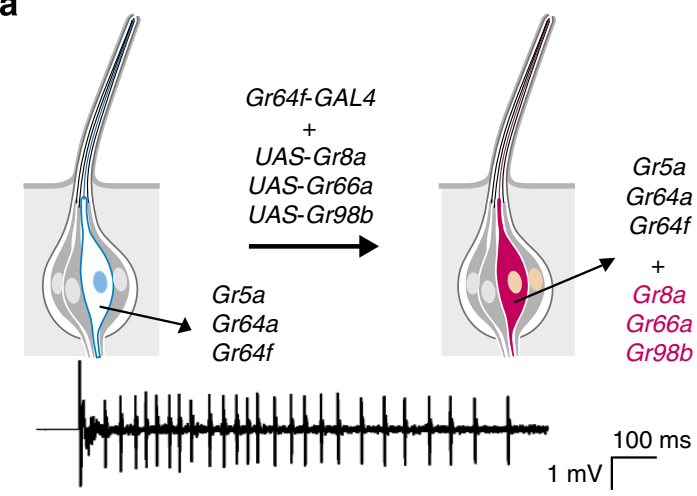

b

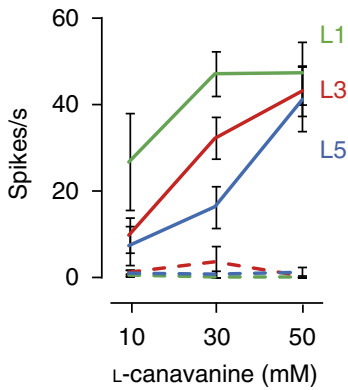

C
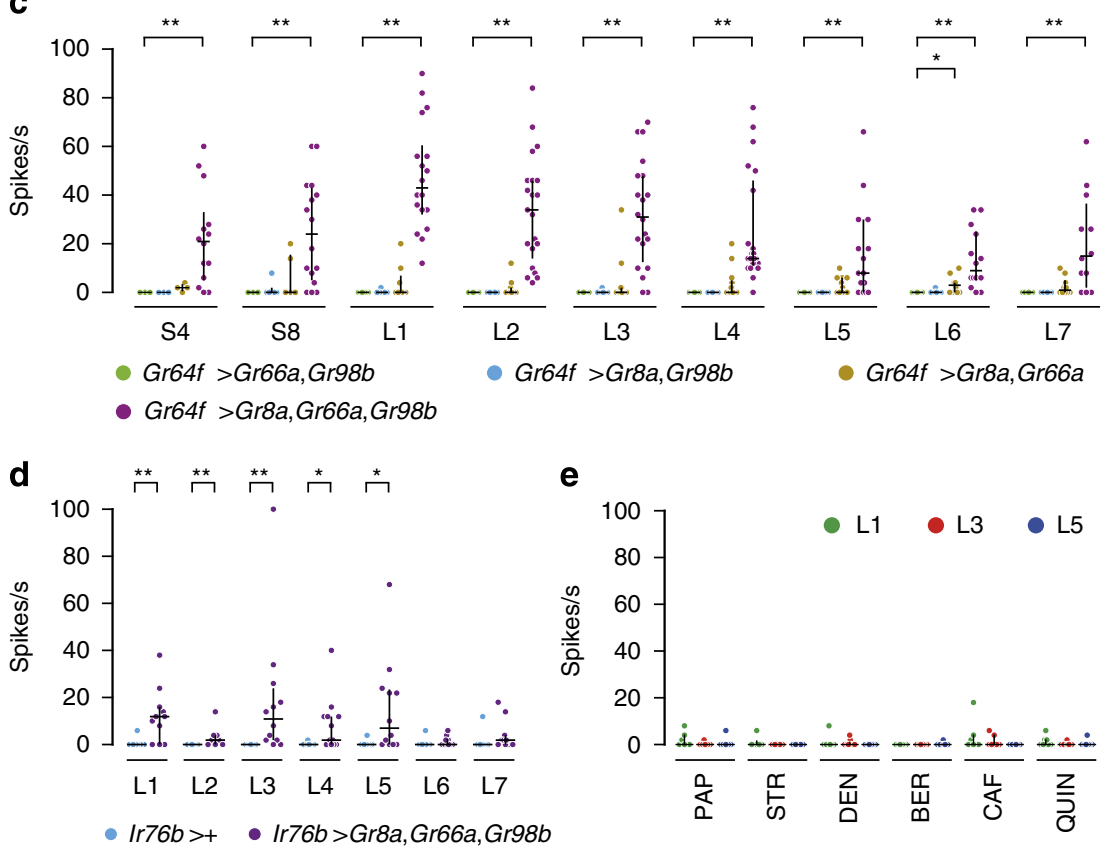

Figure 4 | Effects resulting from ectopic expression of Gr8a, Gr66a and Gr98b in sugar and low-salt responsive GRNs. (a) Schematic depicting ectopic expression of Gr8a, Gr66a and Gr98b in sweet-sensing GRNs (above) and representative traces (below) evoked by 30 mM L-canavanine from L3 sensilla expressing Gr8a, Gr66a and Gr98b in sweet-sensing GRNs. (b) Dose-dependent L-canavanine responses in the indicated sweet-sensing GRNs. Shown are the responses of the indicated sensilla from control flies without a GAL4 (UAS-Gr8a,UAS-Gr66a,UAS-Gr98b; dashed lines) or flies expressing the three Grs under the control of the Gr64f-GAL4 (solid lines). $n=6-21$. Data are mean \pm s.e.m. (c) Response frequencies evoked by $30 \mathrm{mM}$ L-canavanine after ectopic expression of Gr8a, Gr66a and Gr98b in the indicated sweet-sensing GRNs using the Gr64f-GAL4. Genotypes: (1) green circles: $2 \times$ (UAS-Gr66a,UAS-Gr98b);Gr64f-GAL4/ +, (2) blue circles: $2 \times$ (UAS-Gr8a,UAS-Gr98b);Gr64f-GAL4/ +, (3) ochre circles: $2 \times$ (UAS-Gr8a, UAS-Gr66a);Gr64f-GAL4/ + and (4) purple circles: $2 \times\left(\right.$ UAS-Gr8a, UAS-Gr66a,UAS-Gr98b);Gr64f-GAL4/+. $n=4-21$ for each genotype. Shown are the medians and quartiles. ${ }^{\star} P<0.05,{ }^{\star \star} P<0.01$ (KruskalWallis test with Mann-Whitney U post hoc-test). (d) Response frequencies evoked by $30 \mathrm{mM}$ L-canavanine after ectopic expression of Gr8a, Gr66a and Gr98b in the indicated low salt-sensing GRNs using the Ir76b-GAL4. Genotypes: (1) blue circles: Ir76b-GAL4/ +, and (2) purple circles: UAS-Gr8a,UAS-Gr66a,UASGr98b/ +; Ir76b-GAL4/+. $n=8-14$. Median and quartile are shown. ${ }^{\star} P<0.05,{ }^{\star \star} P<0.01$ (Mann-Whitney U-test). (e) Response frequencies from the indicated sensilla in flies ectopically expressing Gr8a, Gr66a and Gr98b in sugar-responsive GRNs. We tested $1 \mathrm{mM}$ of each of the indicated chemicals (BER, berberine; DEN, denatonium; LOB, lobeline; PAP, papaverine; STR, strychnine), except for caffeine (CAF; $5 \mathrm{mM}) . n=6-9$. Median and quartile are shown. 
To determine whether Gr8a, Gr66a and Gr98b were sufficient to confer L-canavanine sensitivity to GRNs that normally do not respond to L-canavanine, we ectopically expressed Gr8a and Gr98b in Gr66a-expressing GRNs that were insensitive to L-canavanine ${ }^{22,30-32}$. Misexpression of Gr8a in Gr66aexpressing, L-canavanine-insensitive GRNs does not confer $\mathrm{L}$-canavanine sensitivity ${ }^{22}$. We found that introduction of Gr8a and Gr98b in L-canavanine-insensitive S2- and I-type sensilla under the control of the Gr33a-GAL4 conferred robust $\mathrm{L}$-canavanine responses to these sensilla (Fig. $3 \mathrm{~b}, \mathrm{c}$ ). These results indicated that GR8a and GR98b were essential components of the functional L-canavanine receptor.

Conferring L-canavanine responsiveness to sweet-sensing GRNs. Since bitter-sensing GRNs in S2- and I-type sensilla also express other GRs ${ }^{30}$, it is possible that additional avoidance GRs may be involved in L-canavanine detection. Wild-type L-type sensilla contain four GRNs, one of which respond to sugars and not to L-canavanine ${ }^{22}$. To provide stronger evidence that Gr8a, Gr66a and Gr98b were sufficient for L-canavanine sensation, we misexpressed these three Grs in sugar-activated GRNs in L-type sensilla, using the Gr64f-GAL4 (Fig. 4a). We found that this manipulation endowed sweet-sensing GRNs with the ability to respond robustly to L-canavanine (Fig. $4 \mathrm{~b}, \mathrm{c}$ ). Ectopic expression of only two of the three GRs was insufficient to confer significant L-canavanine responsiveness (Fig. 4c). However, in 12 out of 90 recordings, co-expression of just two of the Grs (Gr8a and Gr66a) produced a small L-canavanine-evoked response in L-type sensilla (Fig. 4c). We also ectopically expressed Gr8a, Gr66a and Gr98b in low salt-sensing Ir76b-GAL4-positive GRNs ${ }^{33}$, and found that this conferred significant L-canavanine responsiveness to these GRNs (Fig. 4d). Ectopic expression of Gr8a, Gr66a and Gr98b did not induce responsiveness to any of several bitter compounds tested (Fig. 4e).

Eliciting behavioural attraction to L-canavanine. Because expression of Gr8a, Gr66a and Gr98b was sufficient to endow L-canavanine sensitivity to sweet-sensing GRNs, we tested whether ectopic expression of these Grs induced attraction to L-canavanine.

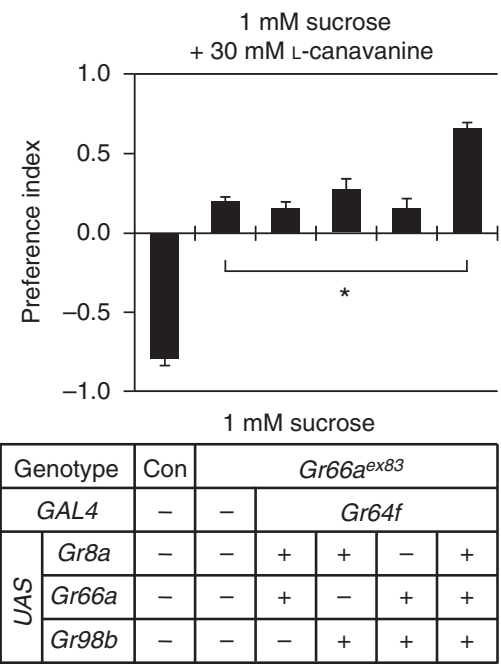

Figure 5 | Attraction to L-canavanine induced by ectopic expression of Grs in sugar-sensing GRNs. Two-way choice assays testing for attraction or aversion to L-canavanine in flies misexpressing Gr8a, Gr66a and Gr98b in sweet-sensing GRNs. The Grs were ectopically expressed in a Gr66a ex83 mutant background. $n=5$ for each genotype. Data are mean \pm s.e.m. ${ }^{\star} P<0.05$ (analysis of variance with post hoc Tukey test).
Control flies $\left(w^{1118}\right)$ strongly preferred $1 \mathrm{mM}$ sucrose over $1 \mathrm{mM}$ sucrose laced with $30 \mathrm{mM}$ L-canavanine (Fig. 5). In contrast, Gr66 $a^{e x 83}$ mutants had no preference for either alternative. $G r 66 a^{e x 83}$ flies expressing just two Grs (Gr8a/Gr66a, Gr8a/Gr98b or Gr66a/Gr98b) in sweet-sensing GRNs were also nearly indifferent to sucrose alone versus sucrose plus L-canavanine (Fig. 5). However, introduction of all three Grs (Gr8a/Gr66a/Gr98b) induced significant attraction to the L-canavanine-containing food (Fig. 5).

GR8a/GR66a/GR98b-dependent L-canavanine current in S2 cells. To test whether GR8a, GR66a and GR98b could form an L-canavanine-activated cation channel, we co-expressed the three GRs in Drosophila S2 cells and performed whole-cell voltage-clamp recordings. We clamped the cells at a holding potential of $-60 \mathrm{mV}$ and applied voltage ramps from -80 to $+80 \mathrm{mV}$. Using a normal physiological bath solution, we found that addition of $30 \mathrm{mM}$ L-canavanine produced a slightly outwardly rectifying current with a reversal potential of $-0.88 \pm 1.89 \mathrm{mV}$ (Fig. 6a,b). However, no current was produced when we expressed any combination of two GRs (GR8a/GR66a, GR8a/GR98b or GR66a/GR98b; Fig. 6c-f). The L-canavanine-induced currents were completely inhibited by $\mathrm{La}^{3+}$, a broad spectrum cation channel inhibitor (Fig. 6g,h). Consistent with the in vivo data, no other aversive compounds tested induced a conductance in S2 cells expressing GR8a, GR66a and GR98b (Fig. 6i).

\section{Discussion}

Perception of toxic compounds and the aversive behaviors they elicit are innate defense mechanisms shared by many animals, including insects. In Drosophila, the detection of most aversive compounds depends on members of the GR family ${ }^{19-23,30}$. The subunit composition of GR receptors is complicated. Consequently, a major challenge in the field has been to define the repertoire of subunits that are sufficient to render a functional bitter receptor in vivo and in vitro.

We and others have made multiple attempts to elucidate the composition of a functional receptor complex that senses a repulsive compound $21,23,30,34$. However, none of these undertakings have been entirely successful. For example, Gr33a, Gr66a and Gr93a are essential for detecting caffeine, but misexpression of these GRs in sweet-sensing GRNs is insufficient to confer sensitivity to caffeine $e^{23}$. Similarly, we have defined several GRs that contribute to DEET sensation by $\mathrm{GRNs}^{19}$. However, ectopic expression of these GRs in non-DEETresponsive GRNs is not adequate to elicit DEET sensitivity ${ }^{19}$. Ectopic expression of Gr59c in Gr21a/Gr63a $\mathrm{CO}_{2}$-sensing neurons or in a subset of bitter-sensing GRNs that do not normally respond to berberine, denatonium and lobeline confers sensitivity to these tastants ${ }^{30,34}$. Nevertheless, GR59c has not been functionally expressed in vitro, and so it remains unclear whether it forms a homomeric or heteromeric receptor. Consistent with the latter possibility, misexpression of $G r 59 c$ in sweet-sensing GRNs is ineffective, indicating that one or more additional GRs are required for detection of these avoidance compounds $s^{30}$.

We found that Gr8a, Gr66a and Gr98b were sufficient to generate a functional L-canavanine-sensing GR complex in vivo in every type of GRN tested that was formerly unresponsive to L-canavanine. These included low salt-sensing and sweet-sensing GRNs. Moreover, activation of sweet GRNs with L-canavanine, after misexpressing the three Grs, converted the natural aversion to L-canavanine to attraction ${ }^{35-37}$. Because low-salt and sweet GRNs do not normally express any aversive GR, our findings indicate strongly that there are no additional GRs that comprise the L-canavanine receptor. 
a

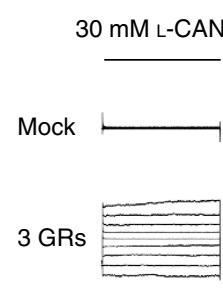

$30 \mathrm{pA} / \mathrm{pF}\llcorner$ $100 \mathrm{~ms}$ b

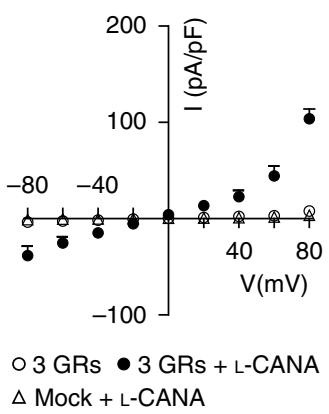

c
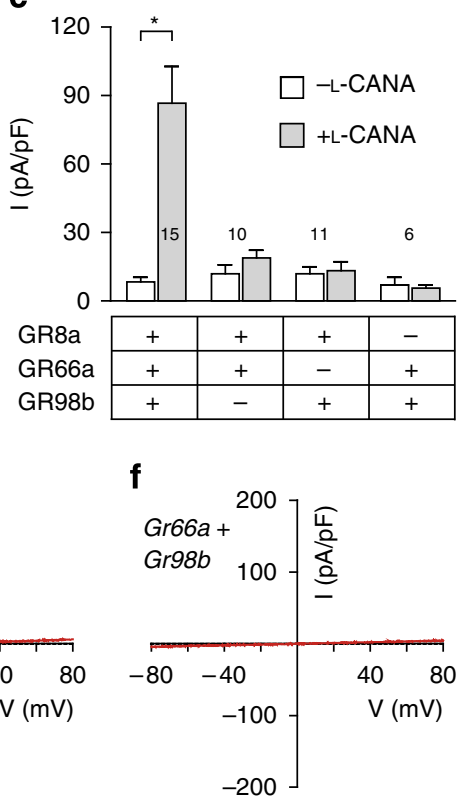

d

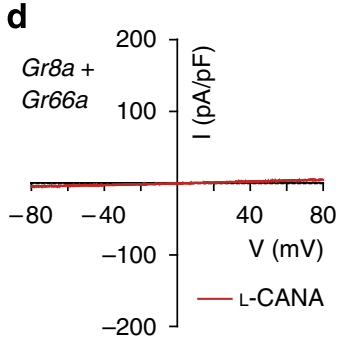

g

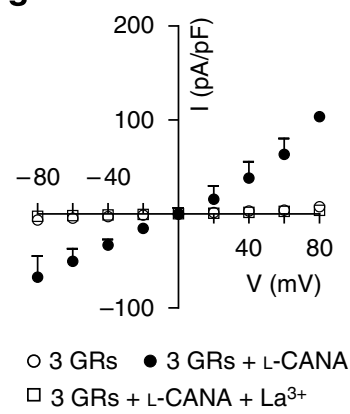

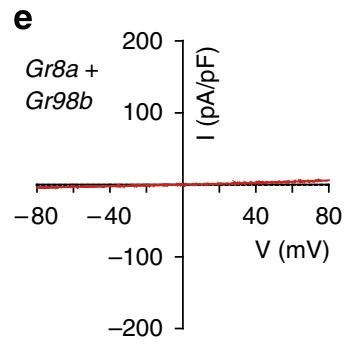

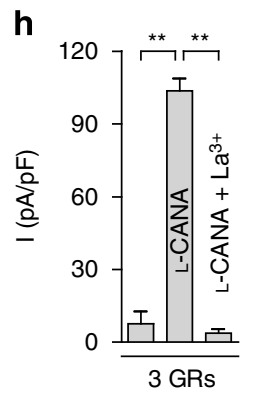

i

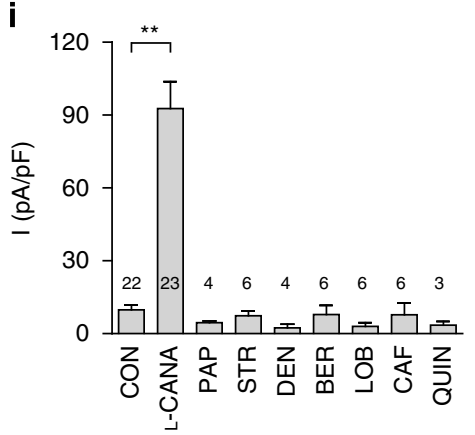

Figure 6 | Whole-cell voltage clamp recordings of S2 cells expressing GRs. Cells were stimulated with 30 mM L-canavanine as indicated. (a,b) Cells were transfected either with pActin5c-GAL4, pUAST-EGFP only (mock) or pActin5c-GAL4, pUAST-EGFP plus pUAST-Gr8a, pUAST-Gr66a and pUAST-Gr98b ( $3 \mathrm{GRs}$ ). (a) Currents produced in response to voltage steps ( $-80 \mathrm{mV}$ to $+80 \mathrm{mV}$ in $20 \mathrm{mV}$ increments) of 500-ms duration obtained in the presence of L-canavanine. (b) I-V relationships using cells expressing GR8a, GR66a and GR98b, and stimulated with L-canavanine. (c) Current densities at +80 mV. The cells expressed the indicated GRs and were recorded in the presence or absence of L-canavanine stimulation. The numbers of recordings are indicated. ${ }^{\star} P<0.05$ (paired Student's $t$-test). (d-f) Current-voltage traces showing that expression of two GRs in S2 cells did not lead to L-canavanine-induced increases in current densities. (d) Gr8a- and Gr66a-expressing S2 cells $(n=10)$. (e) Gr8a- and Gr98b-expressing cells ( $n=11$ ). (f) Gr66a- and Gr98bexpressing cells $(n=6)$. ( $(\mathbf{g})$ Effect of $\mathrm{La}^{3+}$ on I-V relationship. The cells expressed GR8a, GR66a and GR98b, and were recorded in the presence of L-canavanine. (h) Effect of $\mathrm{La}^{3+}$ on current densities obtained at $+80 \mathrm{mV}$. The cells expressed the three GRs and were stimulated with L-canavanine. ${ }^{\star \star} P<0.01$ (analysis of variance (ANOVA) with post hoc Tukey test). (i) Current densities in cells expressing GR8a, GR66a and GR98b and stimulated with the indicated bitter chemicals: $30 \mathrm{mM}$ L-canavanine, $1 \mathrm{mM}$ papaverine (PAP), $1 \mathrm{mM}$ strychnine (STR), $1 \mathrm{mM}$ denatonium (DEN), $100 \mu \mathrm{M}$ berberine (BER), $1 \mathrm{mM}$ lobeline (LOB), $5 \mathrm{mM}$ caffeine (CAF), and $1 \mathrm{mM}$ quinine (QUIN). The numbers of recordings are indicated. ${ }^{\star} P<0.01$ (ANOVA with post hoc Tukey test). All error bars indicate s.e.m.

The combination of GR8a and GR66a resulted in a low level of L-canavanine sensitivity in a few GRNs that do not normally respond to L-canavanine. Thus, GR8a and GR66a may produce a low-affinity L-canavanine receptor, while GR98b is required for a high-affinity receptor. The GR heteromultimers required for sensing other aversive tastants may be even more complex than the three GR subunits that are required and sufficient for responding to L-canavanine. Because ectopic expression of the three GRs known to be required for responding to caffeine or the three GRs essential for tasting DEET are insufficient for conferring responses to these compounds ${ }^{19,21}$, these assemblies appear to be comprised of at least four GRs subunits.

The minimum number of GR subunits critical for sensing attractive tastants remains unresolved. Ectopic expression of single GRs in the $\mathrm{CO}_{2}$-sensing olfactory neurons endows these cells with glycerol and sugar sensitivities, raising the possibility that homomeric GRs elicit these responses ${ }^{34,38}$. However, $\mathrm{CO}_{2}$ neurons express GR21a, GR63a ${ }^{39}$ and possibly other GRs that could potentially form heteromultimeric GR complexes. Mutational analyses and unsuccessful ectopic expression studies indicate that most sugar receptors consist of a complexity of subunits ${ }^{40-42}$. The notable exceptions are insect fructose receptors, which function as ligand-gated cation channels in HEK293 cells ${ }^{18}$.

We demonstrated that introduction of GR8a, GR66a and GR98b in vitro in Drosophila S2 cells conferred an L-canavanine-induced cation conductance. Thus, we conclude that these three GRs comprise a heteromultimeric L-canavanineactivated channel, which is required and sufficient for detecting this aversive compound in GRNs. This three subunit channel is 
more complex than the channels formed by the distantly related ORs, which are comprised of just two subunits ${ }^{15,16}$. One of the two OR subunits is a broadly required olfactory co-receptor, which is essential for trafficking of the OR complex ${ }^{43}$. Similarly, since GR66a is essential for sensing a broad array of aversive tastants ${ }^{19}$, it may also represent a co-receptor that participates in trafficking of other GRs, such as GR8a and GR98b, which provide tastant specificity.

Finally, activation of the L-canavanine cation conductance in GRNs inhibits feeding in Drosophila. Other Dipterans such as mosquitoes feed on human hosts and in doing so, spread prevalent diseases, including malaria and Dengue fever. The discovery that a Drosophila GR receptor complex that responds to a repulsive compound is a cation channel, offers the possibility of finding effective compounds to suppress feeding by insect disease vectors, by performing high-throughput screens for activators of similar complexes that function in mosquitoes.

\section{Methods}

Fly stocks. All fly stocks were maintained on conventional cornmeal-agarmolasses medium, with 12 -h light/12-h dark cycles at $25^{\circ} \mathrm{C}$ and $60 \%$ humidity. We obtained the following fly stocks from the Bloomington Stock Center: (1) 70FLP,70I-SceI/CyO, (2) UAS-Dcr2 and (3) UAS-mCD8::GFP. The UAS-Gr8a, UAS-Gr66a, Gr33a-GAL4 and Gr8a-GAL4 flies were described previously ${ }^{20-22,30}$. The Gr66a-GAL4 flies were a gift from H. Amrein ${ }^{32}$. Gr98b-GAL4 and Gr64fGAL4 were provided by J. Carlson ${ }^{30,44}$. We obtained the fly stocks for the RNA screen from the Vienna Drosophila RNAi Center and the Bloomington Stock Center. The stock numbers are as follows: Gr2a (v102185), Gr5a (v13730), Gr8a (v31104), Gr9a (v15446), Gr10a (v39237), Gr10b (v31151), Gr21a (v104122), Gr22a (v106736), Gr22b (v107792), Gr22c (v7249), Gr22e (v9389), Gr22f (v102860), Gr23a (v40852), Gr28a (v100938), Gr28b (v101727), Gr32a (v47956), Gr33a (v42802), Gr36a (v48018), Gr36b (v8062), Gr36c (v3872), Gr39a (v8685), Gr39b (v33215), Gr43a (v39518), Gr47b (v4594), Gr57a (v45879), Gr58a (v1703), Gr58b (v9565), Gr58c (v29137), Gr59a (v31107), Gr59b (v101219), Gr59c (v3530), Gr59d (v2766), Gr59e (v31110), Gr59f (v18989), Gr61a (v106007), Gr63a (v108203), Gr64a (v103342), Gr64b (v42517), Gr64c (BL36734), Gr64d (v29422), Gr64e (v109176), Gr64f (v105084), Gr66a (v14820), Gr68a (v13380), Gr77a (BL38236), Gr85a (v47992), Gr89a (v8253), Gr92a (v44408), Gr93a (v13569), Gr93b (v12160), Gr93c (v109794), Gr93d (v6813), Gr94a (v9537), Gr97a (v4395), Gr98a (v1300), Gr98b (v1302 and v101040), Gr98c (BL36735), Gr98d (v4398), IR7a (v108171), IR47a (v11812), IR56a (v5010), IR56b (v4704), IR56d (v6112), IR94e (v33066), IR20a (v8658), IR94a (v7566), IR94c (v6817), IR94h (v1563), IR60b (v12089), IR67c (v37261) and IR94f (v109702).

Genetics. We generated the Gr98b mutant $\left(G r 98 b^{1}\right)$ by ends-out homologous recombination. To obtain the DNA construct for the homologous recombination, we used a genomic DNA template from isogenic $w^{1118}$ flies and PCR to amplify $3 \mathrm{~kb}$ arms located $5^{\prime}$ and $3^{\prime}$ to the targeted Gr98b locus. The primers used for the $5^{\prime}$ arm were $5^{\prime}$-GGTGGCTTAGGTGCTGCCATTAC- $3^{\prime}$ and $5^{\prime}$-TTGGGTGAGT TCTGAAAACTAAC- $3^{\prime}$. The primers for the $3^{\prime}$ arm were $5^{\prime}$-TCTGAAACGCAA TCAATTGCTA- $3^{\prime}$ and $5^{\prime}$-GTAGCCCAATATCACAATTC- $3^{\prime}$. We subcloned the two arms into the pw35 vector ${ }^{45}$, the transgenic flies were generated by germline transformation (BestGene, Inc., Chino Hills, CA), and the transgene was mobilized to generate the homologous recombinants as described ${ }^{45}$. We confirmed the $G r 98 b^{1}$ allele via genomic PCR, in conjunction with the following primers: $5^{\prime}$-TCTCCTG GCCAGAGCCTTTCCATA- $3^{\prime}$ and $5^{\prime}$-TGCTGCATTATCATGACGAACTCGG- $3^{\prime}$.

To generate the UAS-Gr98b transgenic flies, we amplified a Gr98b cDNA from a $w^{1118}$-derived labellar cDNA library using the Hi-fidelity PCR kit (Roche), and cloned the cDNA into the pUAST vector. We verified the cDNA clone by DNA sequencing and the transgenic flies were generated by BestGene, Inc. We outcrossed the Gr98b $b^{1}$ and the UAS-Gr98b flies to $w^{1118}$ flies for five generations.

Imaging. We performed immunostaining of whole mount fly labella ${ }^{21}$ using rabbit anti-green fluorescent protein (GFP) (1:1,000, Molecular Probes) primary antibodies and goat anti-rabbit Alexa488 (1:400, Molecular Probes) secondary antibodies. The labella were dissected from heads, fixed for $20 \mathrm{~min}$ using $4 \%$ paraformaldehyde diluted in PBS-T $(1 \times$ PBS for $20 \mathrm{~min}$ and $0.2 \%$ TritonX-100) and washed three times with PBS-T. The labella were bisected with a razor blade, incubated for $30 \mathrm{~min}$ in blocking solution (5\% heat-inactivated goat serum in PBS$\mathrm{T}$ ) and incubated overnight at $4{ }^{\circ} \mathrm{C}$ with the primary antibodies diluted in the blocking solution. The tissues were washed three times with PBS-T and incubated with the secondary antibodies diluted in blocking solution for $1 \mathrm{~h}$ at room temperature. Following three washes with PBS-T, the samples were mounted with Vectashield (Vector Laboratories, Burlingame, CA) and visualized with a Zeiss LSM700 confocal microscope (Jena, Germany).
Chemicals. Sucrose, denatonium, quinine, papaverine, caffeine, strychnine, -canavanine, N,N-diethyl-m-toluamide (DEET), sulforhodamine $\mathrm{B}, \mathrm{KCl}$ and tricholine citrate were purchased from Sigma-Aldrich (Saint Louis, MO). Berberine sulfate trihydrate and Brilliant Blue FCF were obtained from Wako Pure Chemical Industries, Ltd (Osaka, Japan).

Two-way choice behavioural assay. The binary food choice assays were performed in a blinded fashion as described previously ${ }^{11,20}$. Briefly, for each assay we starved $\sim 50$ (3-6 days old) flies for $18 \mathrm{~h}$ and placed them in 72-well microtiter dishes. Each alternating well was filled with $1 \%$ agarose combined with one of the two types of test mixtures. The aversion to bitter chemicals was assayed by comparing the preferences for $1 \mathrm{mM}$ sucrose to $5 \mathrm{mM}$ sucrose plus the indicated concentrations of aversive compounds. To measure the effect of activation of sugar GRNs by L-canavanine, we tested the preference for $1 \mathrm{mM}$ sucrose versus $1 \mathrm{mM}$ sucrose plus $30 \mathrm{mM}$ L-canavanine.

To monitor food intake, we added blue dye (Brilliant Blue FCF, $0.125 \mathrm{mg} \mathrm{ml}^{-1}$ ) to one test mixture, and red dye (sulforhodamine $\mathrm{B}, 0.2 \mathrm{mg} \mathrm{ml}^{-1}$ ) to the other. After allowing the flies to feed for $90 \mathrm{~min}$ at room temperature in the dark, the animals were frozen at $-20^{\circ} \mathrm{C}$. The numbers of blue $\left(\mathrm{N}_{\mathrm{B}}\right)$, red $\left(\mathrm{N}_{\mathrm{R}}\right)$ or purple $\left(\mathrm{N}_{\mathrm{MIX}}\right)$ flies were counted under a dissection microscope and the preference index (PI) values were calculated according to the following equation: $\left(\mathrm{N}_{B}-\mathrm{N}_{\mathrm{R}}\right)$ / $\left(\mathrm{N}_{\mathrm{R}}+\mathrm{N}_{\mathrm{B}}+\mathrm{N}_{\mathrm{MIX}}\right)$ or $\left(\mathrm{N}_{\mathrm{R}}-\mathrm{N}_{\mathrm{B}}\right) /\left(\mathrm{N}_{\mathrm{R}}+\mathrm{N}_{\mathrm{B}}+\mathrm{N}_{\mathrm{MIX}}\right)$. PIs of 1.0 and -1.0 indicate complete preference for one or the other food. A PI of 0 indicates no preference.

Tip recordings. Tip recordings ${ }^{46}$ were performed as we described previously ${ }^{20}$ Following eclosion, we maintained flies on fresh food for 1 day. We immobilized the animals by inserting a glass capillary reference electrode filled with Ringer's solution into the abdomen, and extending it to the head. We stimulated labellar sensilla with recording electrodes $(10-20 \mu \mathrm{m}$ tip diameter) containing tastants dissolved in $1 \mathrm{mM} \mathrm{KCl}$ or $30 \mathrm{mM}$ tricholine citrate. The recording electrode was connected to a preamplifier (TastePROBE, Syntech, Hilversum, The Netherlands) and taste responses were collected and amplified $(10 \times)$ using a signal interface (Syntech) in conjunction with a $100-3,000 \mathrm{~Hz}$ band-pass filter. The inputs were also linked to a loudspeaker to facilitate audio monitoring. We recorded action potentials at a $12-\mathrm{kHz}$ sampling rate, sorted the spikes based on amplitude, and performed quantification using Autospike 3.1 software package (Syntech).

Cell culture and transfection. We grew S2 cells in Schneider's Insect media (Welgene, Gyeongsan-si, Republic of Korea) supplemented with $10 \%$ fetal bovine serum (Invitrogen, Carlsbad, CA), 50 units per ml penicillin-streptomycin (Invitrogen) in T-25 flasks (Thermo, Waltham, MA) at $25^{\circ} \mathrm{C}$. To perform the patch clamp experiments, we transfected cells $24 \mathrm{~h}$ after plating with pActin $5 \mathrm{c}-G A L 4$, pUAST-EGFP (enhanced green fluorescent protein) and the two or three of the following $G r$ plasmids using X-tremeGENE HP DNA transfection reagent (Roche): pUAST-Gr8a, pUAST-Gr66a and pUAST-Gr98b. The transfection mixture consisted of $4 \mu \mathrm{l}$ of transfection reagent and $1.3 \mu \mathrm{g}$ of total DNA. After incubating the cells with the transfection cocktail in serum-free media for $12 \mathrm{~h}$, we switched to serum-containing media, and continued to incubate the cells for $24 \mathrm{~h}$ to allow for expression of the GRs and EGFP.

Patch clamp experiments in $\mathbf{S 2}$ cells. We transferred $G r$ - and EGFP-expressing S2 cells on coverslips to a chamber positioned on the stage of an inverted microscope (IX71, Olympus). Whole cell currents were measured using an Axon 200B amplifier at a holding potential of $-60 \mathrm{mV}$. The bath solution contained normal Ringer's solution (in $\mathrm{mM}$ ): $140 \mathrm{NaCl}, 5 \mathrm{KCl}, 5 \mathrm{HEPES}, 2$ pyruvic acid sodium salt $1.25 \mathrm{KH}_{2} \mathrm{PO}_{4}, 2 \mathrm{CaCl}_{2}, 2 \mathrm{MgCl}_{2}$ and $10 \mathrm{D}$-glucose (pH 7.4). The pipette solution contained (in $\mathrm{mM}$ ): $140 \mathrm{KCl}, 5$ EGTA-2 K, 10 HEPES and $10 \mathrm{D}$-glucose (pH 7.2). We pulled electrodes from borosilicate glass that had resistances of $2-4 \mathrm{M} \Omega$ after fire polishing. The seal resistances were between 3 and $10 \mathrm{G} \Omega$. After establishing a whole-cell configuration, we recorded currents in the presence of $\mathrm{L}$-canavanine, by applying hyperpolarizing and depolarizing voltage pulses using a holding potential of $-60 \mathrm{mV}$ and voltage ramps from +80 and $-80 \mathrm{mV}$ in steps of $20 \mathrm{mV}$. We performed all recordings at room temperature using an Axopatch-200B amplifier (Axon Instruments, Foster City, CA). We digitized the currents with a Digidata $1440 \mathrm{~A}$ converter (Axon Instruments) filtered at $5 \mathrm{kHz}$. Command potential and data acquisition were controlled with pClamp 10.2 software (Axon instruments). Whole-cell recording data analyses were performed using Clampfit 10.2. The current densities were normalized to the cell capacitance.

Statistical analyses. We performed statistical analyses using the SPSS 21.0 (IBM Corporation, Armonk, NY). All data, except for ectopic expression of GRs, were analysed using unpaired Student's $t$-tests for comparing two sets of data or one-way analysis of variance with Tukey post hoc tests for comparing multiple sets of data, as these data passed the Kolmogorov-Smirnov test. The data represent the means \pm s.e.m.

Electrophysiological data for misexpression of Grs were analysed using nonparametric tests (Figs $2 \mathrm{c}$ and $3 \mathrm{c}$,d). We employed the Mann-Whitney $U$-test for comparing two sets of data. We performed a Kruskal-Wallis test with MannWhitney $U$ post hoc-test to determine whether the medians of two genotypes were significantly different. The data presented are the medians and quartiles. 


\section{References}

1. Liman, E. R., Zhang, Y. V. \& Montell, C. Peripheral coding of taste. Neuron 81, 984-1000 (2014).

2. Kim, S. H. et al. Drosophila TRPA1 channel mediates chemical avoidance in gustatory receptor neurons. Proc. Natl Acad. Sci. USA 107, 8440-8445 (2010).

3. Kwon, Y. et al. Drosophila TRPA1 channel is required to avoid the naturally occurring insect repellent citronellal. Curr. Biol. 20, 1672-1678 (2010).

4. Zhang, Y. V., Raghuwanshi, R. P., Shen, W. L. \& Montell, C. Food-experience induced taste desensitization modulated by the Drosophila TRPL channel. Nat. Neurosci. 16, 1468-1476 (2013).

5. Clyne, P. J., Warr, C. G. \& Carlson, J. R. Candidate taste receptors in Drosophila. Science 287, 1830-1834 (2000).

6. Dunipace, L., Meister, S., McNealy, C. \& Amrein, H. Spatially restricted expression of candidate taste receptors in the Drosophila gustatory system. Curr. Biol. 11, 822-835 (2001).

7. Robertson, H. M., Warr, C. G. \& Carlson, J. R. Molecular evolution of the insect chemoreceptor gene superfamily in Drosophila melanogaster. Proc. Natl Acad. Sci. USA 100(Suppl 2): 14537-14542 (2003).

8. Scott, K. et al. A chemosensory gene family encoding candidate gustatory and olfactory receptors in Drosophila. Cell 104, 661-673 (2001).

9. Clyne, P. J. et al. The odor specificities of a subset of olfactory receptor neurons are governed by Acj6, a POU-domain transcription factor. Neuron 22, 339-347 (1999).

10. Gao, Q. \& Chess, A. Identification of candidate Drosophila olfactory receptors from genomic DNA sequence. Genomics 60, 31-39 (1999).

11. Meunier, N., Marion-Poll, F., Rospars, J. P. \& Tanimura, T. Peripheral coding of bitter taste in Drosophila. J. Neurobiol. 56, 139-152 (2003).

12. Montell, C. A taste of the Drosophila gustatory receptors. Curr. Opin. Neurobiol. 19, 345-353 (2009).

13. Scott, K. The sweet and the bitter of mammalian taste. Curr. Opin. Neurobiol. 14, 423-437 (2004)

14. Vosshall, L. B., Amrein, H., Morozov, P. S., Rzhetsky, A. \& Axel, R. A spatial map of olfactory receptor expression in the Drosophila antenna. Cell 96, 725-736 (1999).

15. Sato, K. et al. Insect olfactory receptors are heteromeric ligand-gated ion channels. Nature 452, 1002-1006 (2008).

16. Wicher, D. et al. Drosophila odorant receptors are both ligand-gated and cyclicnucleotide-activated cation channels. Nature 452, 1007-1011 (2008).

17. Turner, R. M. et al. Mutational analysis of cysteine residues of the insect odorant co-receptor (Orco) from Drosophila melanogaster reveals differential effects on agonist- and odorant-tuning receptor-dependent activation. J. Biol. Chem. 289, 31837-31845 (2014).

18. Sato, K., Tanaka, K. \& Touhara, K. Sugar-regulated cation channel formed by an insect gustatory receptor. Proc. Natl Acad. Sci. USA 108, 11680-11685 (2011).

19. Lee, Y., Kim, S. H. \& Montell, C. Avoiding DEET through insect gustatory receptors. Neuron 67, 555-561 (2010).

20. Moon, S. J., Köttgen, M., Jiao, Y., Xu, H. \& Montell, C. A taste receptor required for the caffeine response in vivo. Curr. Biol. 16, 1812-1817 (2006).

21. Moon, S. J., Lee, Y., Jiao, Y. \& Montell, C. A. Drosophila gustatory receptor essential for aversive taste and inhibiting male-to-male courtship. Curr. Biol. 19, 1623-1627 (2009).

22. Lee, Y. et al. Gustatory receptors required for avoiding the insecticide L-canavanine. J. Neurosci. 32, 1429-1435 (2012).

23. Lee, Y., Moon, S. J. \& Montell, C. Multiple gustatory receptors required for the caffeine response in Drosophila. Proc. Natl Acad. Sci. USA 106, 4495-4500 (2009).

24. Lee, Y., Moon, S., Wang, Y. \& Montell, C. A Drosophila gustatory receptor required for strychnine sensation. Chem. Senses 40, 525-533 (2015).

25. Dahlman, D. L. \& Rosenthal, G. A. Non-protein aminoacid-insect interactionsI. Growth effects and symptomology of L-canavanine consumption by tobacco hornworm, Manduca sexta (L.). Comp. Biochem. Physiol. A Comp. Physiol. 51, 33-36 (1975).

26. Rosenthal, G. A. L-Canavanine: a higher plant insecticidal allelochemical. Amino Acids 21, 319-330 (2001).

27. Rosenthal, G. A. \& Dahlman, D. L. L-Canavanine and protein synthesis in the tobacco hornworm Manduca sexta. Proc. Natl Acad. Sci. USA 83, 14-18 (1986).

28. Croset, V. et al. Ancient protostome origin of chemosensory ionotropic glutamate receptors and the evolution of insect taste and olfaction. PLoS Genet. 6, e1001064 (2010).

29. Koh, T. W. et al. The Drosophila IR20a clade of ionotropic receptors are candidate taste and pheromone receptors. Neuron 83, 850-865 (2014).

30. Weiss, L. A., Dahanukar, A., Kwon, J. Y., Banerjee, D. \& Carlson, J. R. The molecular and cellular basis of bitter taste in Drosophila. Neuron 69, 258-272 (2011).

31. Wang, Z., Singhvi, A., Kong, P. \& Scott, K. Taste representations in the Drosophila brain. Cell 117, 981-991 (2004).
32. Thorne, N., Chromey, C., Bray, S. \& Amrein, H. Taste perception and coding in Drosophila. Curr. Biol. 14, 1065-1079 (2004).

33. Zhang, Y. V., Ni, J. \& Montell, C. The molecular basis for attractive salt-taste coding in Drosophila. Science 340, 1334-1338 (2013).

34. Freeman, E. G., Wisotsky, Z. \& Dahanukar, A. Detection of sweet tastants by a conserved group of insect gustatory receptors. Proc. Natl Acad. Sci. USA 111, 1598-1603 (2014).

35. Marella, S. et al. Imaging taste responses in the fly brain reveals a functional map of taste category and behavior. Neuron 49, 285-295 (2006).

36. Mueller, K. L. et al. The receptors and coding logic for bitter taste. Nature 434, 225-229 (2005).

37. Zhao, G. Q. et al. The receptors for mammalian sweet and umami taste. Cell 115, 255-266 (2003).

38. Wisotsky, Z., Medina, A., Freeman, E. \& Dahanukar, A. Evolutionary differences in food preference rely on Gr64e, a receptor for glycerol. Nat. Neurosci. 14, 1534-1541 (2011).

39. Jones, W. D., Cayirlioglu, P., Grunwald Kadow, I. \& Vosshall, L. B. Two chemosensory receptors together mediate carbon dioxide detection in Drosophila. Nature 445, 86-90 (2007).

40. Jiao, Y., Moon, S. J. \& Montell, C. A Drosophila gustatory receptor required for the responses to sucrose, glucose, and maltose identified by mRNA tagging. Proc. Natl Acad. Sci. USA 104, 14110-14115 (2007).

41. Jiao, Y., Moon, S. J., Wang, X., Ren, Q. \& Montell, C. Gr64f is required in combination with other gustatory receptors for sugar detection in Drosophila. Curr. Biol. 18, 1797-1801 (2008).

42. Slone, J., Daniels, J. \& Amrein, H. Sugar receptors in Drosophila. Curr. Biol. 17, 1809-1816 (2007).

43. Benton, R., Sachse, S., Michnick, S. W. \& Vosshall, L. B. Atypical membrane topology and heteromeric function of Drosophila odorant receptors in vivo. PLoS Biol. 4, e20 (2006).

44. Dahanukar, A., Lei, Y. T., Kwon, J. Y. \& Carlson, J. R. Two Gr genes underlie sugar reception in Drosophila. Neuron 56, 503-516 (2007).

45. Gong, W. J. \& Golic, K. G. Ends-out, or replacement, gene targeting in Drosophila. Proc. Natl Acad. Sci. USA 100, 2556-2561 (2003).

46. Hodgson, E. S., Lettvin, J. Y. \& Roeder, K. D. Physiology of a primary chemoreceptor unit. Science 122, 417-418 (1955).

\section{Acknowledgements}

We thank H. Amrein and J. Carlson for providing reagents, and the Bloomington Stock Center and the Vienna Drosophila RNAi Center for providing fly stocks. We thank Takaaki Sokabe for performing some of the initial patch clamp experiments. J.S. was supported by the Korea Food Research Institute, Republic of Korea. This study was supported by a grant to S.J.M. from the Basic Science Research Program through the National Research Foundation of Korea (NRF) funded by the Ministry of Education, Science and Technology (NRF-2012R1A1A1012081 and NRF-2014R1A2A1A11050045), a grant to M.G.L. from the National Research Foundation, the Ministry of Science, ICT \& Future Planning, Republic of Korea (2013R1A3A2042197), a grant to Y.L. from a new faculty research program (J2013-0027) of Kookmin University in Korea and a grant to C.M. from the National Institute on Deafness and Other Communication Disorders (DC007864).

\section{Author contributions}

Y.L. conducted the Gr RNAi screen and performed the tip recordings shown in Figs 1 and 2. J.S. performed most of the genetic studies. Y.T.J. conducted the electrophysiology analysis. Y.K. and M.G.L. performed patch clamp experiments. C.M. and S.J.M. supervised the project and wrote the manuscript.

\section{Additional information}

Supplementary Information accompanies this paper at http://www.nature.com/ naturecommunications

Competing financial interests: The authors declare no competing financial interests.

Reprints and permission information is available online at http://npg.nature.com/ reprintsandpermissions/

How to cite this article: Shim, J. et al. The full repertoire of Drosophila gustatory receptors for detecting an aversive compound. Nat. Commun. 6:8867 doi: $10.1038 /$ ncomms 9867 (2015).

This work is licensed under a Creative Commons Attribution 4.0 International License. The images or other third party material in this article are included in the article's Creative Commons license, unless indicated otherwise in the credit line; if the material is not included under the Creative Commons license, users will need to obtain permission from the license holder to reproduce the material. To view a copy of this license, visit http://creativecommons.org/licenses/by/4.0/ 